\title{
Synthesis of novel functional liquid and its application as a modifier in SBR/silica composites
}

\author{
Y. D. Lei $^{1}$, Z. H. Tang ${ }^{1}$, B. C. Guo ${ }^{1,2^{*}}$, L. X. Zhu', D. M. Jia ${ }^{1}$ \\ ${ }^{1}$ Department of Polymer Materials and Engineering, South China University of Technology, Guangzhou 510640, China \\ ${ }^{2}$ State Key Laboratory of Pulp and Paper Engineering, South China University of Technology, Guangzhou 510640, China
}

Received 18 May 2010; accepted in revised form 13 July 2010

\begin{abstract}
A novel functional ionic liquid (IL), 1-methylimidazolium methacrylate (MimMa), was synthesized for modifying styrene butadiene rubber (SBR)/silica composites. MimMa was found to be readily polymerized via the initiated radical mechanism and could be analogously grafted onto rubber chains during vulcanization. Substantial hydrogen bonding between polymerized MimMa (poly(MimMa)) and silica can facilitate the silica dispersion and improve the SBR/silica interfacial bonding. Filler networking, curing behavior, silica dispersion and mechanical performance of the modified $\mathrm{SBR} /$ silica composites were studied. With a low concentration of MimMa, remarkable improvements in the interfacial interactions and mechanical properties were achieved which was attributed to the improved silica dispersion and strengthened interfacial bonding induced by MimMa. A modified interphase structure was accordingly proposed and related to the mechanical performance of the modified SBR/silica composites.
\end{abstract}

Keywords: rubber, silica, ionic liquid, interface

\section{Introduction}

The filler dispersion and the rubber/filler interfacial bonding are two critical factors in determining the performance of the filled rubber composites. Conventionally, the surface treatments with various modifiers such as silanes have been used to improve the filler dispersion and strength the rubber/silica interfacial bonding [1-4]. Due to the high polarity of silica particles, the dispersion of silica in silane included systems and controllability of the performance are far from satisfactory. Ionic liquid (IL), a kind of fascinating molten salt with nearly zero vapor pressure and high thermal stability [5-7], has demonstrated high affinity toward various kinds of solid, such as carbon nanotubes [8-14], silica [15-17] and clays [18]. Various interactions, including cation- $\pi$ interaction $[8-11,19]$, van der Waal forces [13], delocalized electron interaction [14] and hydrogen bonding [16, 18] have been well acknowledged and therefore subjected to a plenty of applications [14, 16-18, 20-26]. Thanks to these interactions, ILs with specific structure can be employed as possible interfacial modifiers for various filled polymer composites. However, due to the limited compatibility toward rubber [27-29] and the environmental unfriendliness of the fluorine containing anions [30], these two factors deter the common ILs such as 1-butly-3-methylimidazole hexafluorophosphate $\left(\mathrm{BmimPF}_{6}\right)$ from their corresponding promising applications in modifying rubber/filler composites. Previously, we have confirmed that, via special treating methods, such as solution dispersion, mechanical grinding or microwave irradiation treatment, $\mathrm{BmimPF}_{6}$ could not only influence surface properties of rubber fillers, but also improve the mechanical performance of the filled polymer materials [31-33]. However, an excess loading of 
$\mathrm{BmimPF}_{6}$ proved to be unacceptable because of its poor interaction toward rubber chains and its high economic cost. Consequently, designing functional ILs with special functional structure and economic rationality is of crucial importance in exploring novel interfacial modifier for rubber/filler composites.

In a recent study, protic ILs could be high efficiently fabricated and their structure ccould be easily tailored with numerous commercially available raw materials such as Bronsted acids and bases [34-38]. In our previous work, a functional group was successfully induced into the anion of IL. Through a procedure similar to the synthesis of protic ILs, 1-methylimidazolium sorbate (MimS) was synthesized and investigated as a modifier for improving silica dispersion and interfacial bonding [39]. The mechanical performance of the MimS modified styrene butadiene rubber (SBR)/silica composites were effectively improved. In the present work, another novel functional IL, 1-methylimidazolium methacrylate (MimMa), is synthesized and utilized as a modifier for the SBR/silica rubber composites. Based on the disclosed MimMa/silica interaction and the graftability of MimMa toward rubber chains, an improved interfacial structure is proposed. The performance of SBR/silica composites is studied. The vulcanization behavior, mechanical performance and the abrasion resistance are all correlated to the improved dispersion and interfacial structure induced by MimMa.

\section{Experimental}

\subsection{Materials}

$\mathrm{N}$-methylimidazole, with $99 \%$ purity, was purchased from Alfa Aesar, TianJin, China. Methacrylic acid, analytical grade, was produced by Tianjin Bodi Chemical Holding Co. Ltd, China. Styrene butadiene rubber (SBR), with trade name SBR1502 (styrene content $23.5 \mathrm{wt} \%$ ), was manufactured by Jilin Chemical Industry Company, China.

Precipitated silica, with trade name WL180, was manufactured by NanPing Jialian Chem. Ltd., China. The Brunauer-Emmett-Teller (BET) value of silica was re-determined to be $200 \mathrm{~m}^{2} / \mathrm{g}$ by Micromeretics ASAP 2020. Other rubber additives were industrial grade and used as received.

\subsection{Synthesis of MimMa}

Stoichiometric N-methylimidazole was added dropwise to methacrylic acid. The reaction was kept below $20^{\circ} \mathrm{C}$ under stirring and lasted for 1 hour. The transparent colorless liquid product (1-methylimidazolium methacrylate, MimMa) was characterized by FTIR spectroscopy (Bruker Vertex 70 FTIR spectrometer) and ${ }^{1} \mathrm{H}$ NMR spectroscopy (Bruker AVANCE Digital NMR spectrometer, $300 \mathrm{MHz}) .{ }^{1} \mathrm{H} \mathrm{NMR}\left(\mathrm{CDCl}_{3}, 300 \mathrm{MHz}\right)$ : $11.83(\mathrm{~s}, 1 \mathrm{H}, \mathrm{N}-\mathrm{H}), 7.68(\mathrm{~s}, 1 \mathrm{H}, \mathrm{N}-\mathrm{CH}=\mathrm{N})$, $7.08-7.09(\mathrm{t}, 1 \mathrm{H}, \mathrm{J} 1=1.20 \mathrm{~Hz}, \mathrm{~J} 2=1.05 \mathrm{~Hz}$, $\mathrm{C}=\mathrm{CH}-\mathrm{N}=\mathrm{C}), \quad 6.86-6.87 \quad(\mathrm{t}, \quad 1 \mathrm{H}, \mathrm{J}=1.29 \mathrm{~Hz}$, $\left.\left(\mathrm{CH}_{3}\right) \mathrm{N}-\mathrm{CH}=\mathrm{C}-\mathrm{N}\right), \quad 6.11-6.13 \quad(\mathrm{~m}, \quad 1 \mathrm{H}, \quad$ cis $-\mathrm{OOC}-\mathrm{C}=\mathrm{CH}$ ), 3.69 (s, 3H, N-CH 3$), 1.94-1.95$ $\left(\mathrm{m}, 3 \mathrm{H},\left(\mathrm{CH}_{3}\right)(-\mathrm{OOC}) \mathrm{C}=\mathrm{C}\right)$. FTIR (Bruker Vertex 70, $\mathrm{KBr}$ ) [40-43]: $3400(\mathrm{~N}-\mathrm{H}), 3130$ (imidazole$\mathrm{H}), 1523(\mathrm{C}=\mathrm{N}), 1693(\mathrm{C}=\mathrm{O}), 1630(\mathrm{C}=\mathrm{C}$ in the anion).

\subsection{Polymerizability of MimMa and its graftability toward SBR chains}

Bulk polymerization of MimMa were conducted with 2,2'-azobisisobutyronitrile (AIBN) (0.5 wt $\%)$ as the initiator at $65^{\circ} \mathrm{C}$ for 4 hours under nitrogen protection. Grafting copolymerization of MimMa onto SBR was performed in toluene with AIBN as the initiator (SBR/MimMa/AIBN, 10/5/0.15, $w t / w t / w t)$. The reaction was conducted at $65^{\circ} \mathrm{C}$ for $5 \mathrm{~h}$. The crude graft product was first washed with deionized water for three times and then extracted with water on a Soxhlet extractor for 3 days. The MimMa grafted product (poly(SBR-g-MimMa)) was then obtained after vacuum drying. Poly(MimMa) and poly(SBR-g-MimMa) were verified by FTIR spectroscopy.

\subsection{Interactions of MimMa and poly(MimMa) with silica}

Another bulk polymerization of MimMa in the presence of silica was performed according to the procedure mentioned above. FTIR, differential scanning calorimetry (DSC; Q5000 differential scanning calorimeter, TA Instruments) and X-ray photon spectroscopy (XPS; Kratos Axis Ultra DLD) were performed to reveal the interaction 
between them. In the XPS experiment, silica and MimMa/silica were tested with a monochromated Aluminum $\mathrm{K} \alpha$ source $(1486.6 \mathrm{eV})$. Those XPS spectra of $\mathrm{Si}$ and $\mathrm{C}$ atoms were obtained by fine scanning with step width of $0.1 \mathrm{eV}$. The MimMa/ silica $(10 / 1, w t / w t)$ sample were mixed, washed with acetone, centrifuged and lastly obtained after a vacuum drying. All XPS spectra were calibrated to its reference $\mathrm{C} 1 \mathrm{~s}$ component at $285.0 \mathrm{eV}$ [44]. A Gaussian function was employed to fit the curve in order to distinguish the chemical components with different chemical environments. The DSC cures on poly(MimMa) and poly(MimMa)/silica were scanned from -85 to $20^{\circ} \mathrm{C}$ at $10^{\circ} \mathrm{C} \cdot \mathrm{min}^{-1}$ with nitrogen as the purging gas.

\subsection{Preparation of SBR/silica compounds and their characterizations}

All the rubber compositions were summarized in Table 1. SBR and other additives were mixed on an open two-mill moll. The dependence of shear modulus $\left(G^{\prime}\right)$ on the strain of uncured rubber compounds was measured with the strain from 0.5 to $100 \%$ at $70^{\circ} \mathrm{C}$ on viscoelastography rubber processing analyzer (Göttfert-Werkstoff-Prüfmaschinen $\mathrm{GmbH}$, Germany). The frequency was set as $1 \mathrm{~Hz}$. The curing characteristics of the rubber compounds were determined by a UR-2030 vulcameter (U-CAN, Taiwan) at $170^{\circ} \mathrm{C}$. After the rubber compounds were vulcanized for vulcanization time $\left(T_{c 90}\right)$ at $170^{\circ} \mathrm{C}$, tensile and tear tests of the vulcanizates were performed according to ISO 37-2005 and ISO 34-2004, respectively. The dynamic mechanical analysis (DMA) was performed on the EPLEXOR dynamic mechanical analyzer (Gabo Qualimeter Testanlagen GmbH; Ahlden, Germany). The samples were scanned from -100 to $100^{\circ} \mathrm{C}$ at $3^{\circ} \mathrm{C} / \mathrm{min}$. A tensile mode was adopted. Scanning electron microscopy (SEM) observation was performed on cryogenically fractured vulcanizates with a LEO 1530 VP scanning electron microscope (Leo system GmbH; Schwerte, Ger- many). Before the observation, a thin gold was evaporated on the fractured surface. Transition electron microscopy (TEM) observations were done on ultramicrotomed slices $(\sim 200 \mathrm{~nm})$ using a Philips Tecnai 12 transmission electron microscope (Eindhoven, Netherlands) with an accelerating voltage of $30 \mathrm{kV}$.

\section{Results and discussion}

\subsection{Polymerization of MimMa and its reactivity towards SBR chains}

Polymerizability of MimMa and its graftability toward SBR chains are characterized by their FTIR spectra (Figure 1). According to the spectra of poly(MimMa), the stretching of the double bound $(\mathrm{C}=\mathrm{C})$ was not found, which indicates the polymerization of MimMa happened. Also a shift of the $\mathrm{C}=\mathrm{O}$ band from 1693 to $1680 \mathrm{~cm}^{-1}$ indicates an environmental change of the anion. In the FTIR spectrum pattern of poly(SBR-g-MimMa), the aromatic hydrogen (3020 and $3060 \mathrm{~cm}^{-1}$ ), the $\mathrm{CH}_{2}$ (2912 and $\left.2843 \mathrm{~cm}^{-1}\right)$, the trans $\mathrm{C}=\mathrm{CH}\left(964 \mathrm{~cm}^{-1}\right)$ and the phenyl $\left(694 \mathrm{~cm}^{-1}\right)$ can be assigned to the SBR backbone [43, 45]. The characteristic groups of MimMa, such as $\mathrm{C}=\mathrm{N}\left(1530 \mathrm{~cm}^{-1}\right)$, carbonyl $\left(1690 \mathrm{~cm}^{-1}\right)$ and $\mathrm{C}=\mathrm{CH}\left(3142 \mathrm{~cm}^{-1}\right)$ in the imida-

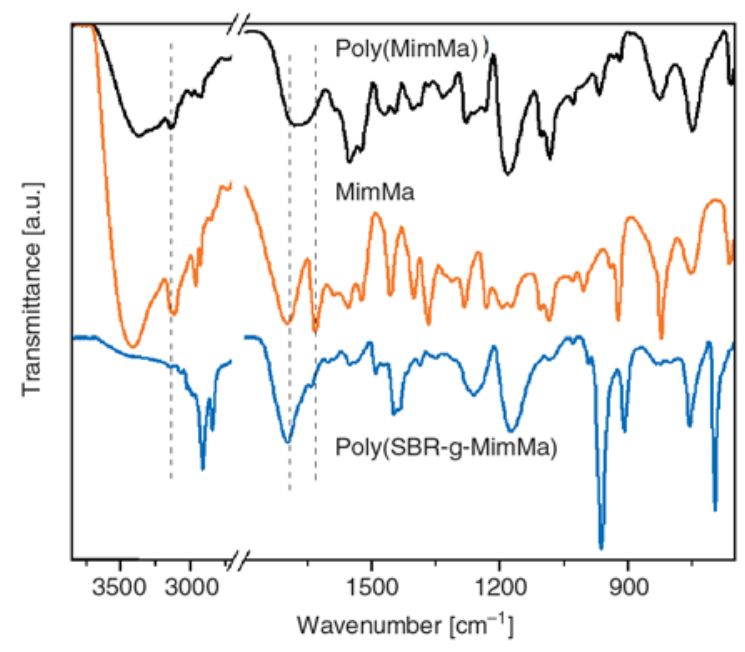

Figure 1. FTIR spectra of MimMa, poly(MimMa) and poly(SBR-g-MimMa)

Table 1. Composition of SBR/silica rubber composites ${ }^{\text {a }}$

\begin{tabular}{|l|c|c|c|c|c|}
\hline \multicolumn{1}{|c|}{ Sample code } & R0MimMa & R1MimMa & R2MimMa & R3MimMa & R4MimMa \\
\hline SBR & 100 & 100 & 100 & 400 \\
\hline Silica & 40 & 40 & 40 & 40 \\
\hline MimMa & 0 & 1 & 2 & 3 \\
\hline
\end{tabular}

a rubber ingredients: zinc oxide 5, stearic acid 1, dicumyl peroxide (DCP) 1,2-mercaptobenzoimidazole 1.5 (all in parts per hundred parts of rubber [phr]) 
zolium cation, indicate the successful grafting of MimMa onto SBR chains.

\subsection{MimMa/silica interactions and its influences on filler networking}

As shown in the upper curve in Figure 2, the binding energies (BE) of silicon ( $\mathrm{Si}$ ) in silanol group $(\mathrm{Si}-\mathrm{OH})$ and in silicon-oxygen bond $(\mathrm{Si}-\mathrm{O}-\mathrm{Si})$ were located at 105.0 and $103.7 \mathrm{eV}$, respectively [46]. Once silica was treated with MimMa, the adsorption peaks of $\mathrm{Si}$ are distinguished. The peak at $103.6 \mathrm{eV}$ was related to the region where is geometrically unavailable for the $\mathrm{Si}-\mathrm{O}-\mathrm{Si} / \mathrm{MimMa}$ interaction. The peak located at $104.0 \mathrm{eV}$ was ascribed to the Si atoms in different original chemical environments. First, it was partially originated from $\mathrm{Si}-\mathrm{OH}$ because the anion of MimMa, interacting with $\mathrm{Si}-\mathrm{OH}$ via hydrogen bonding, could decrease the BE value of Si by strengthening the electron screening effect. Second, it was partially ascribed to the $\mathrm{Si}-\mathrm{O}-\mathrm{Si}$ bond since its $\mathrm{BE}$ value of Si could be increased by weakening its electron screening effect due to the hydrogen bonding between silica and the imidazolium cation.

Due to the disclosed interaction between MimMa and silica, filler networking in rubber compounds, which is usually evaluated by the Payne effect [47, 48], may be influenced a lot. According to the dependence of shear modulus $\left(G^{\prime}\right)$ on strains, the discrepancy of $G^{\prime}\left(\Delta G^{\prime}\right)$ between a small strain $(\sim 0.5 \%)$ and a large strain $(\sim 100 \%)$ can be employed to study the filler networking in the rubber matrix. As a larger extent of the network forms in the filled rubber compounds, a bigger $\Delta G^{\prime}$ appears. The

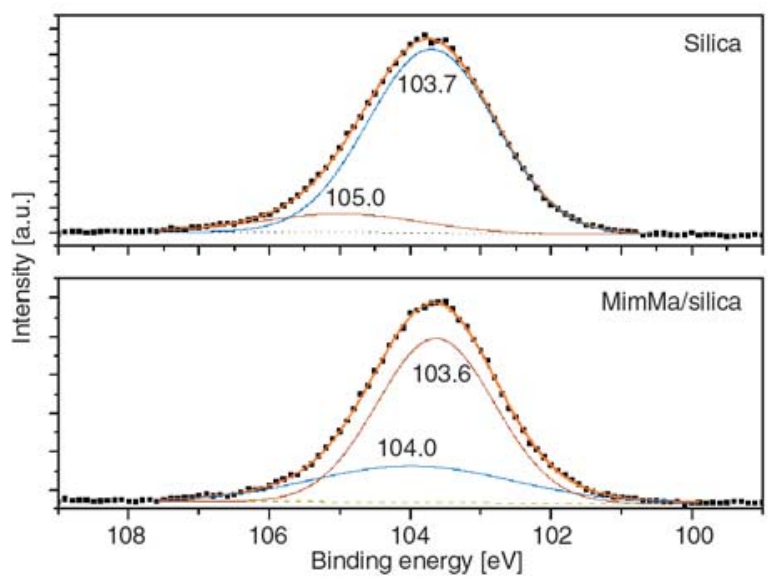

Figure 2. Curve fitting of XPS spectra of Si 2p in silica and poly(MimMa)/silica

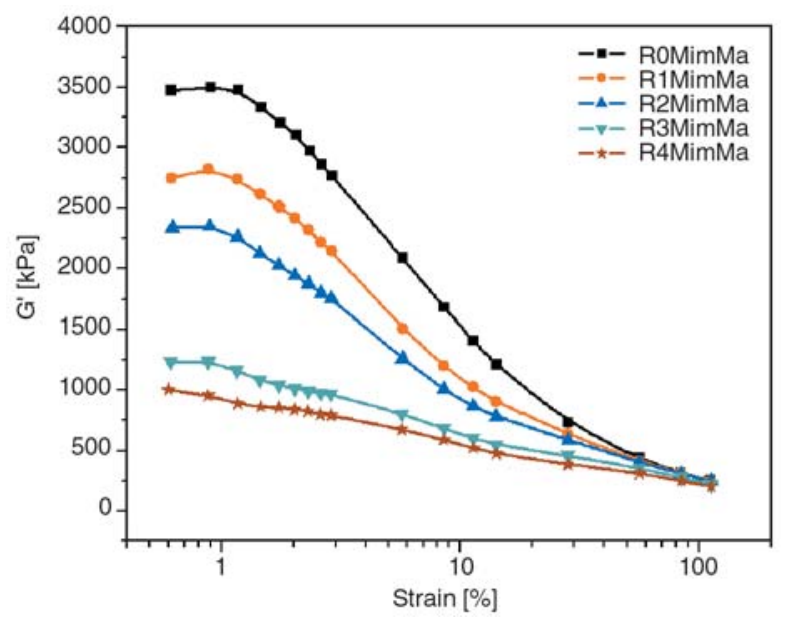

Figure 3. Strain dependence of $\mathrm{G}^{\prime}$ of SBR/silica compounds

dependence of $G^{\prime}$ on strain for the uncured rubber compounds is showed in Figure 3. The value of $G^{\prime}$ at low strains $(<1 \%)$ is high, indicating the formation of silica networking in the rubber matrix. However, with increasing loading of MimMa, the values of $G^{\prime}$ are considerably decreased. For example, the values of $G^{\prime}$ at lower strains $(<1 \%)$ is largely decreased from $3500 \mathrm{kPa}$ for R0MimMa to $1000 \mathrm{kPa}$ for R3MimMa. Furthermore, the values of $\Delta G^{\prime}$ for the rubber compounds are effectively decreased, indicating the weakened Payne effects. Take R4MimMa for example, its values of $\Delta G^{\prime}$ $800 \mathrm{kPa}$, which is only about one quarter of that value of R0Mimma (3220 kPa). The alleviated networking of silica by the addition of MimMa can be ascribed to the weakened interactions among silica particles due to the hydrogen bonding between MimMa and silica as substantiated above.

\subsection{Interactions between poly(MimMa) and silica}

As shown in Figure 1, abundant hydrogen bond functionalities such as carbonyl and imidazole rings are contained in poly(MimMa). Thus, it is expected that poly(MimMa) can interact with silica through special interactions. The FTIR spectra of poly(MimMa) and poly(MimMa)/silica is compared in Figure 4. Red shifts of $\mathrm{N}-\mathrm{H}$ group (from 3364 to $3387 \mathrm{~cm}^{-1}$ ) and $\mathrm{C}=\mathrm{O}$ group (from 1680 to $1693 \mathrm{~cm}^{-1}$ ) were found, while the $\mathrm{C}=\mathrm{C}-\mathrm{H}$ in the imidazolium cation and the $\mathrm{C}-\mathrm{O}$ in the anion showed blue shifts from 3142 to $3122 \mathrm{~cm}^{-1}$ and from 1182 to $1171 \mathrm{~cm}^{-1}$, respectively. Both shifts 


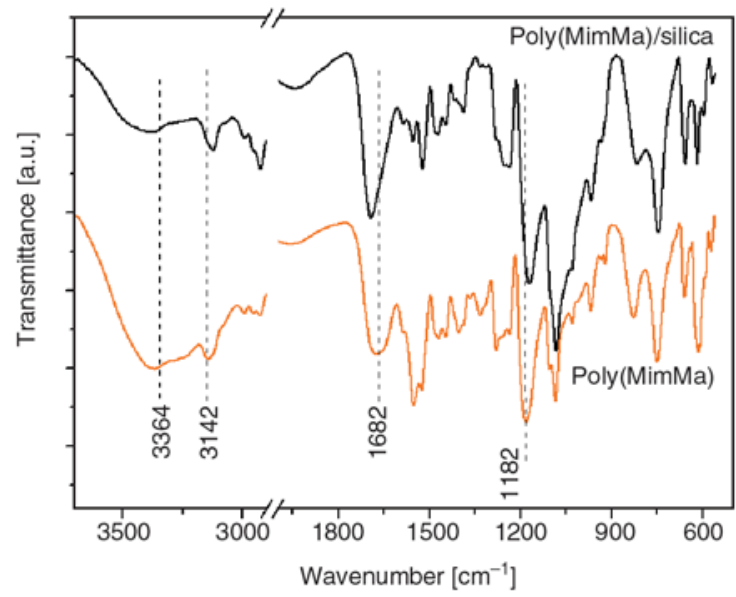

Figure 4. FTIR spectra of poly(MimMa) and poly(MimMa)/silica

strongly suggest that the strong interactions between poly(MimMa) and silica also exist. The possible interaction is partially ascribed to the hydrogen bonding between the imidazolium cation and $\mathrm{Si}-\mathrm{O}-\mathrm{Si}$ and partially ascribed to the hydrogen bonding between the anion and $\mathrm{Si}-\mathrm{OH}$ groups on silica surface, which is well agreement with others' work [16].

The hydrogen bonding may be between poly (MimMa) and silica may affect the glass transition as the mobility of poly(MimMa) was restricted. The DSC curves on poly(MimMa) and poly (MimMa)/silica were presented in Figure 5. The glass transition temperature $\left(T_{g}\right)$ of poly(MimMa) is located around $-57^{\circ} \mathrm{C}$. When about $10 \mathrm{wt} \%$ silica is incorporated, no visible glass transition is detected below $0^{\circ} \mathrm{C}$. It indicates that the mobility of the poly(MimMa) chains is greatly restricted by the strong hydrogen bonding between poly(MimMa) and silica.

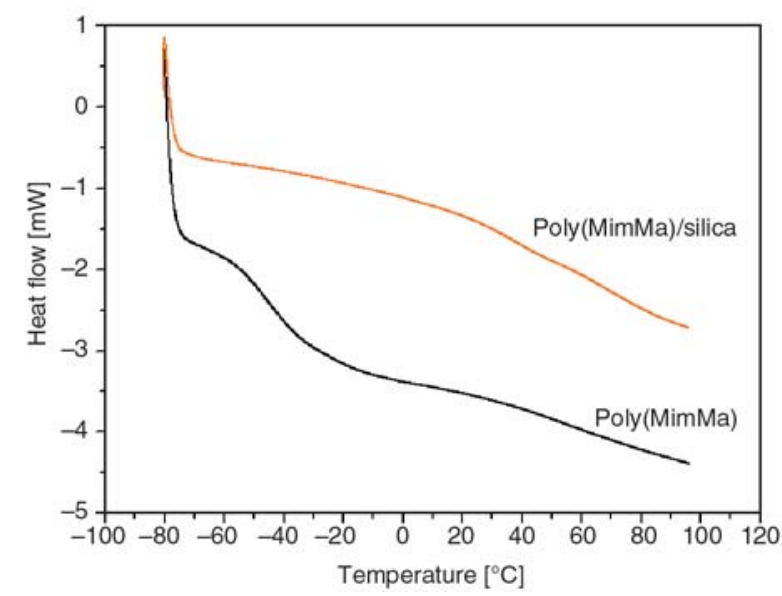

Figure 5. DSC curves for poly(MimMa) and poly(MimMa)/silica

\subsection{Effect of MimMa on the vulcanization behavior of SBR rubber compounds}

Due to the MimMa/filler interaction and the reactivity of MimMa toward SBR which have been substantiated, the vulcanization behavior of the modified SBR/silica rubber compounds was studied. The curing curves are depicted in Figure 6. It can be seen that with higher MimMa loading, the value of the minimum torque $\left(T_{\min }\right)$ during vulcanization is gradually lowered. As indicated from the restrained filler networking in Figure 6, the effective silica volume is substantially decreased because of the weakened interaction among silica particles [49-51]. So with increasing loading of MimMa, the value of $T_{\min }$ is consistently lowered. Interestingly, the maximum torque $\left(T_{\max }\right)$ for these samples does not exhibit a simple trend. With increasing loading of MimMa, the value of $T_{\max }$ takes a maximum for R1MimMa and then gets decreased significantly. There are several factors in governing this change. First, the incorporated MimMa may cover the silica surface and suppress the DCP adsorption on silica. Second, MimMa consumes part of DCP during the vulcanization. Third, the addition of MimMa facilitates the dispersion of silica in rubber and results in lower effective filler volume $[47,52,53]$. The overall effect of MimMa depends upon the competition of these factors. When the first factor dominates, the $T_{\max }$ value will be higher. However, when the last two factors dominate, the $T_{\max }$ value will be lower. When $1 \mathrm{phr}$ of MimMa is used, the consumption of DCP by MimMa and the change in silica dispersion should be limited. So the first factor dominates and higher $T_{\max }$ is observed. When the content of MimMa is

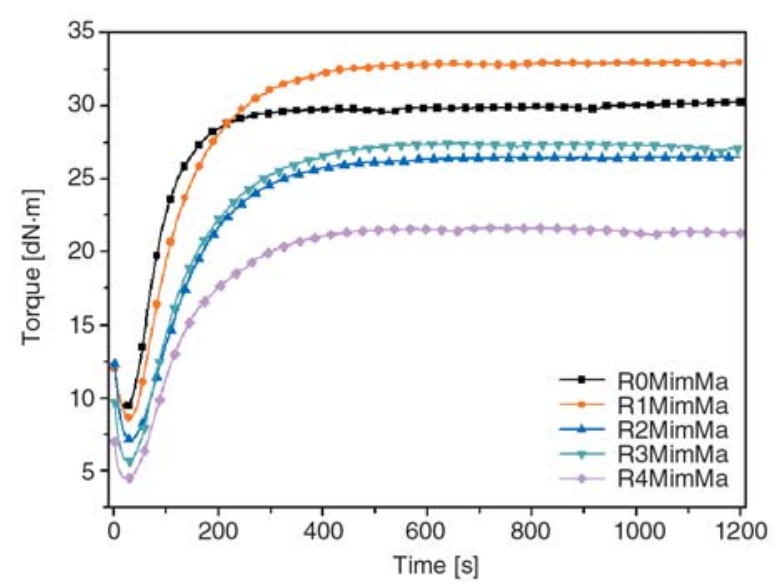

Figure 6. Vulcanization curves of SBR/silica rubber compounds 
further increased, the second and third factors cannot be ignored and consequently much lower $T_{\max }$ values are obtained.

\subsection{Effect of MimMa on the silica dispersion}

Silica dispersion in the rubber matrix is studied by SEM and TEM observations. For ROMimMa vulcanizate, as shown in the two left photos in Figure 7, there are a lot of agglomerates with large sizes more than $100 \mathrm{~nm}$. From these two photos of R4MimMa on the right, it is clear to see that the agglomerate size is significantly decreased and the particles of silica are uniformly dispersed in the rubber matrix. Both the SEM and TEM observations reveal that the dispersion of silica in the modified vulcanizates is obviously improved. The improved filler dispersion is also attributed to the

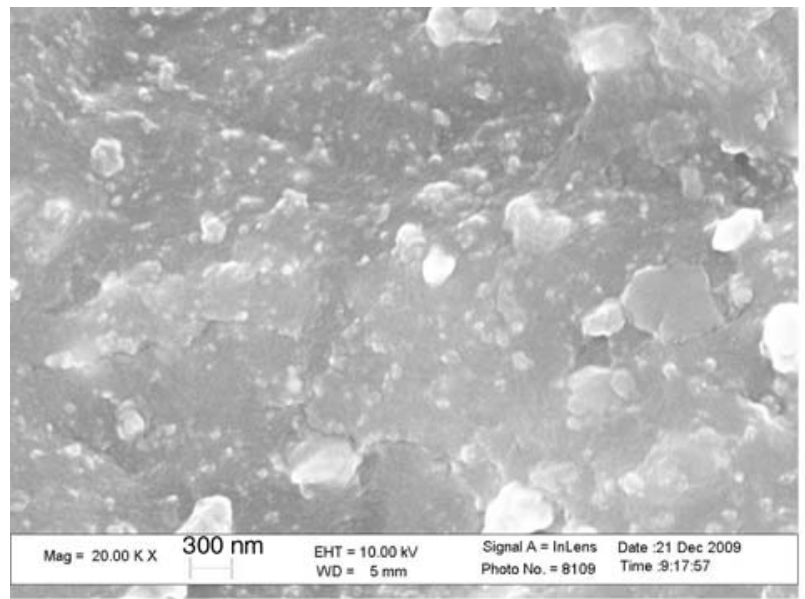

a)

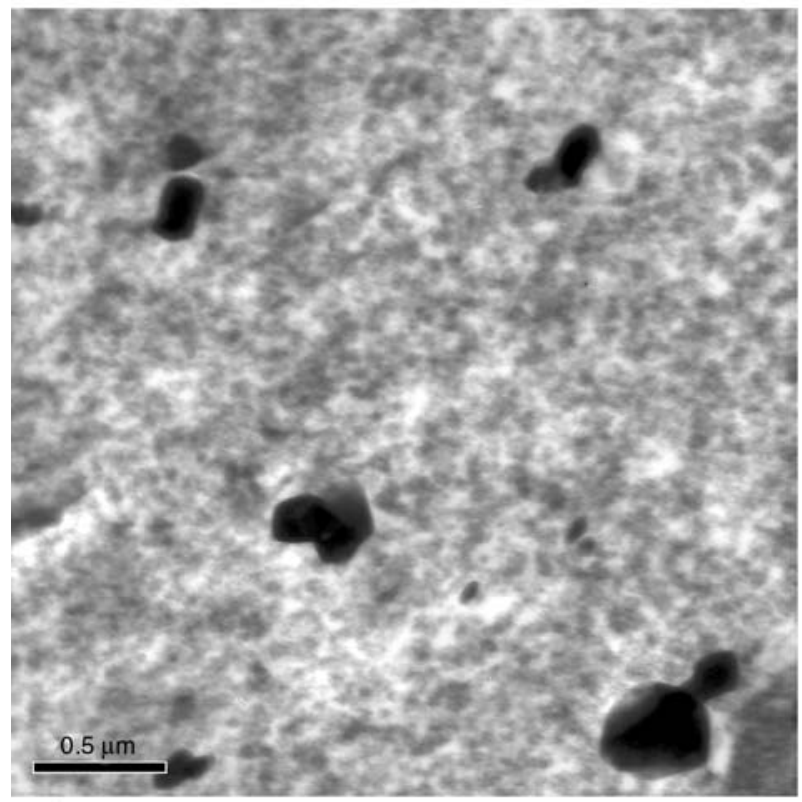

c) interfacial interactions induced by the functional IL. Also, the morphology observations are well consistent with the conclusion derived from the decreased Payne effect in the uncured rubber compounds.

\subsection{Mechanical performance and abrasion resistance of $\mathrm{SBR} /$ silica vulcanizates}

The dependences of tensile strength and tear strengths on MimMa loading are revealed in Table 2 . With only 4 phr of MimMa, the tensile strength is largely increased by an increment of $59 \%$ and the tear strength is enhanced by $58 \%$. Simultaneously, the modulus (always estimated by the stress at $300 \%$ ) is effectively increased by up to $80 \%$. The hardness of the vulcanizates shows practically no variation. From Table 2, the abrasion

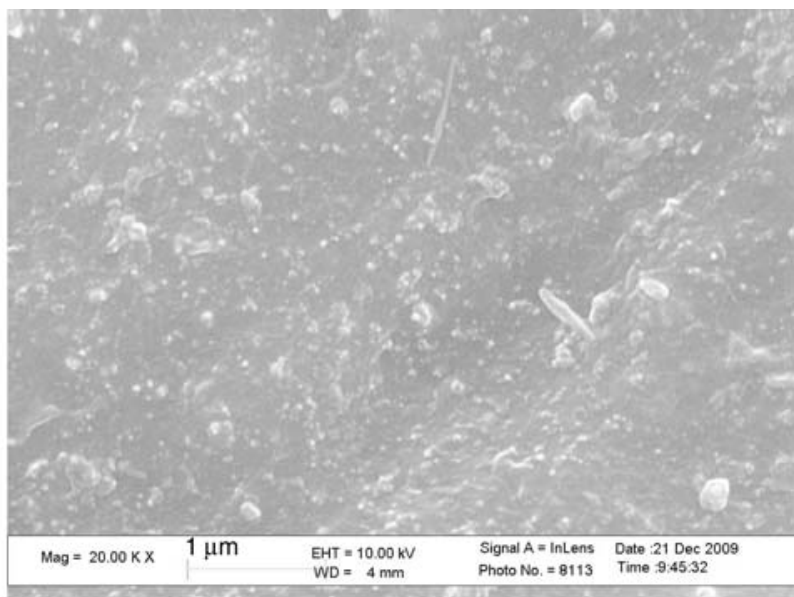

b)

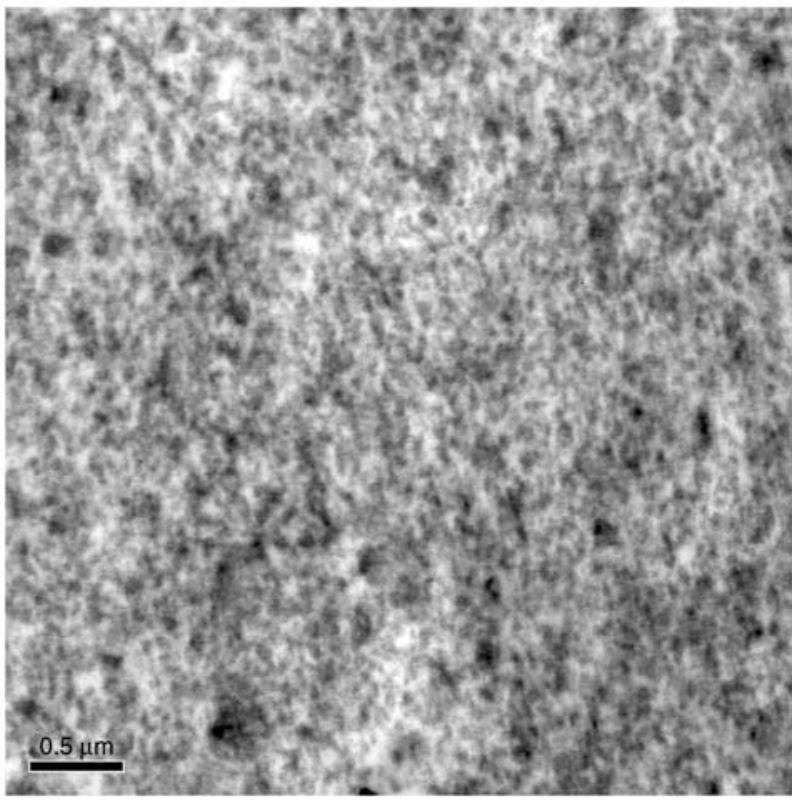

d)

Figure 7. SEM (a, b) and TEM (c, d) photos of R0MimMa and R4MimMa vulcanizates 
Table 2. Mechanical performance of the SBR/silica vulcanizates

\begin{tabular}{|l|c|c|c|c|c|}
\hline & R0MimMa & R1MimMa & R2MimMa & R3MimMa & R4MimMa \\
\hline Stress at 100\% [MPa] & $2.0 \pm 0.1$ & $2.7 \pm 0.1$ & $2.6 \pm 0.1$ & $2.8 \pm 0.1$ & $2.6 \pm 0.1$ \\
\hline Stress at 300\% [MPa] & $4.9 \pm 0.1$ & $7.8 \pm 0.2$ & $7.3 \pm 0.4$ & $9.5 \pm 0.2$ & $8.9 \pm 0.2$ \\
\hline Elongation at break [\%] & $544 \pm 12$ & $436 \pm 12$ & $506 \pm 14$ & $498 \pm 14$ & $589 \pm 15$ \\
\hline Tensile stress [MPa] & $15.4 \pm 1.0$ & $13.9 \pm 1.0$ & $18.9 \pm 0.8$ & $19.5 \pm 1.0$ & $24.5 \pm 1.2$ \\
\hline Tear strength $\left[\mathrm{kN} \cdot \mathrm{m}^{-1}\right]$ & $34.8 \pm 0.5$ & $44.8 \pm 1.6$ & $47.7 \pm 1.8$ & $49.4 \pm 2.5$ & $54.9 \pm 1.2$ \\
\hline Shore A hardness & 69 & 72 & 67 & 68 & 68 \\
\hline Volume loss $\left[\mathrm{cm}^{3} \cdot 1.61 \mathrm{~km}^{-1}\right]$ & 2.86 & 2.68 & 2.27 & 1.85 & 1.56 \\
\hline
\end{tabular}

resistance is also observed. It can be seen that with increasing MimMa loading, the abrasion resistance is consistently improved. The abrasion volume for the R4MimMa is dramatically dropped to $1.56 \mathrm{~cm}^{3} \cdot 1.61 \mathrm{~km}^{-1}$, which is about half of the control one $\left(2.86 \mathrm{~cm}^{3} \cdot 1.61 \mathrm{~km}^{-1}\right)$. All the largely improved performances may be related to the substantiated improved silica dispersion and the interfacial bonding [54], which have been disclosed.

The dynamic mechanical analysis (DMA) of the modified vulcanizates was also performed. The dependence of modulus and tangent delta on temperature is revealed in Figure 8. The glass transition temperature $\left(T_{g}\right)$, indicated from tangent delta in Figure $8 \mathrm{~b}$, is practically unchanged (both around $-50^{\circ} \mathrm{C}$ ). As shown in Figure 8a, the elastic modulus $\left(E^{\prime}\right)$ below $T_{g}$ of R4MimMa is higher than that of R0MimMa. While the loss modulus $\left(E^{\prime \prime}\right)$ of R4MimMa is slightly lowered. Both indicate that the elasticity of the modified vulcanizate below $T_{g}$ is fortified. It may be due to the weakened silica networking and modified interfacial structure in the SBR/silica composites. However, when the sample is in its high elastic state, no practical difference is observed because in this state the mechanical modulus is mainly dependent on the rubber matrix. Noticeably, the mechanical loss of R4MimMa (Figure $8 \mathrm{~b}$ ) is much higher than the control one. It may

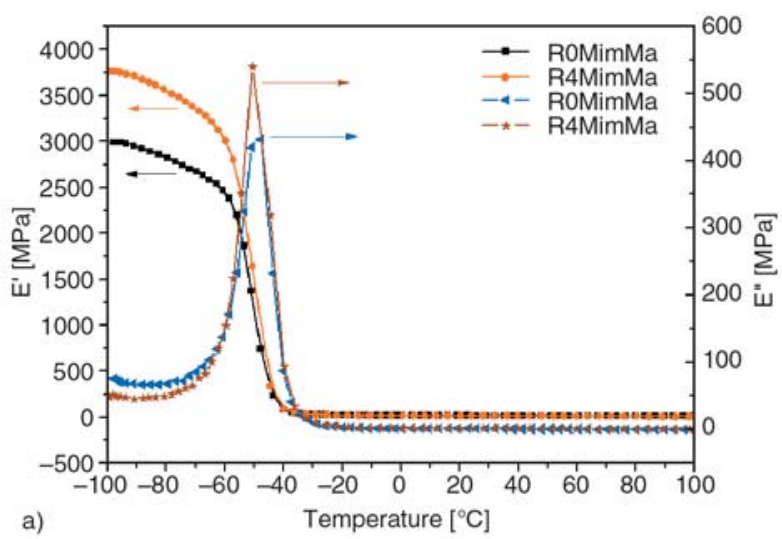

be related to the interfacial structure with noncovalent bondings such as ionic bond or hydrogen bond. It may provide a promising perspective on designing damping material based on the functionality of ILs.

\subsection{Interfacial interactions and proposed modification mechanism}

Within various methods for evaluating the interfacial interactions with the stress-strain curve, the slopes at certain strains and/or certain ranges of strains have always been utilized [55-57]. However, ignoring the significant impact of crosslink density $\left(V_{r}\right)$ on the modulus may be not sound [57]. According to the theory of rubber elasticity, stress of unfilled rubber vulcanizate $\left(\sigma_{r}\right)$ can be theoretically expressed by following.

Considering the ending segments which did not contribute to the rubber network formation but negatively influence the stress at certain strains, $\sigma_{r}$ could be expressed by Equation (1) [58, 59]:

$\sigma_{r}=N_{r} R T\left(\lambda-\frac{1}{\lambda^{2}}\right)$

where $R$ is the universal gas constant, $\lambda$ is the tensile ratio, $T$ is the absolute temperature, $N_{r}$ is the

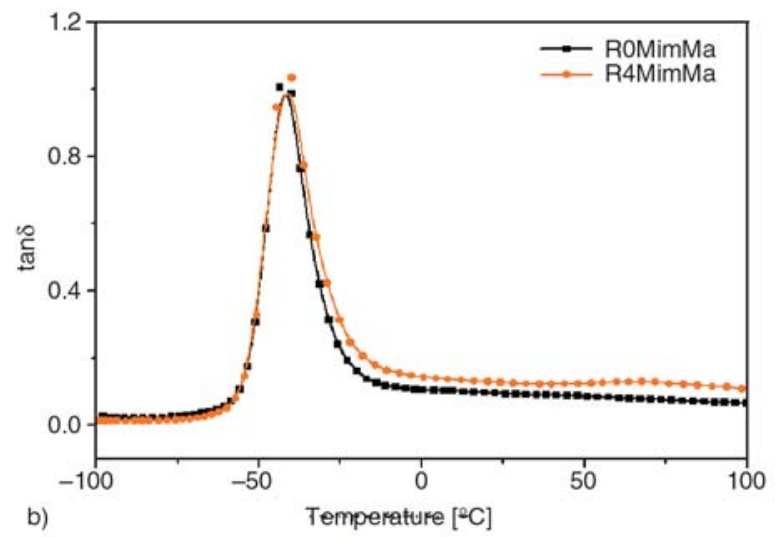

Figure 8. Effect of MimMa on elastic modulus (a) and hysteresis (b) of SBR/silica vulcanizates 
density of chain segments introduced by curatives and also introduced by entanglements of rubber chains.

Stress in filled vulcanizates $\left(\sigma_{c o m}\right)$ was greatly influenced by multi factors including filler, rubber and the interfacial interactions. A possible Equation (2) could be employed to describe $\sigma_{c o m}$ as follows:

$\sigma_{\text {com }}=\psi\left(\sigma_{r}, \sigma_{f}, \sigma_{i n t}\right)$

where $\sigma_{r}, \sigma_{f}$ and $\sigma_{\text {int }}$ are the stress resulting from rubber network, filler and rubber/filler interfacial phase, respectively. The contributions of these three components to $\sigma_{c o m}$ are dependent on strains. Under lower strains, $\sigma_{f}$, which is possibly related to the formation of filler network, has a dominant impact on $\sigma_{c o m}$. However, for the poor deformability of rigid filler network, its contribution to $\sigma_{c o m}$ at large strains may be ignored. Thus, at large strains, the strong interaction between rubber chains and filler was regarded as physical and/or chemical crosslinks [60]. Equation (2) could be approximated by the following Equation (3):

$\sigma_{\text {com }}=\left(N_{r}+N_{\text {int }}\right) R T\left(\lambda-\frac{1}{\lambda^{2}}\right)$

where $N_{\text {int }}$ is the density of chain segments additionally introduced by the interfacial interaction, respectively.

In the calculation of $N_{r}, V_{r}$ was determined by the equilibrium swelling method. $N_{r}$ could be expressed by the product of $V_{r}$ and its functionality of the crosslink points $\left(X_{r}\right)$. Supposed that functionality of crosslink point was 3 and $X_{r}$ was therefore to be $3 / 2$

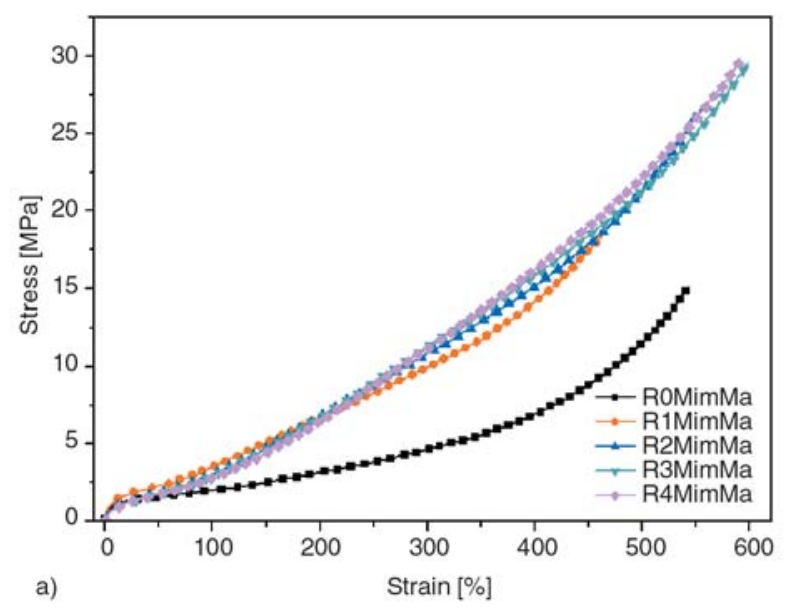

in present system, Equation (3) could be rearranged into Equation (4):

$$
\begin{aligned}
N_{\text {int }} & =\left[\frac{\sigma_{\text {com }}}{R T\left(\lambda-\lambda^{-2}\right)}-N_{r}\right]= \\
& =\frac{\sigma_{c o m}}{R T\left(\lambda-\lambda^{-2}\right)}-\frac{3}{2} V_{r}
\end{aligned}
$$

$N_{\text {int }}$ could thus be utilized to evaluate the interfacial interaction between rubber chains and filler.

Keeping in mind that $N_{r}$ is largely dependent on the deformation of the filled vulcanizates, curves of $N_{\text {int }}$ versus strain of SBR/silica vulcanizates were plotted in Figure 9. Within a range of lower strains, the dramatically high value of $N_{\text {int }}$ may be ascribed to the silica network formation. With increasing strains, the value of $N_{i n t}$ was gradually increased, implying that the SBR/silica interaction was dependent on the deformation of rubber network. Also with increasing strains, stronger interfacial interactions could be observed possibly due to the intensified entanglements and the non Gaussian distribution of the stretched chains. With increasing loading of MimMa as shown in Figure 9, $N_{\text {int }}$ was obviously increased. This strongly indicates that the interfacial interaction between rubber and silica was largely improved. It may be related to the graft copolymerization and the high affinity between MimMa and silica as described above.

Accordingly, an interfacial structure was proposed for explaining the possible mechanism in the present system and elucidated in Figure 10. The hydrogen bonding between MimMa and silica can greatly decrease the Payne effect in uncured rubber compounds. The filler networking is obviously

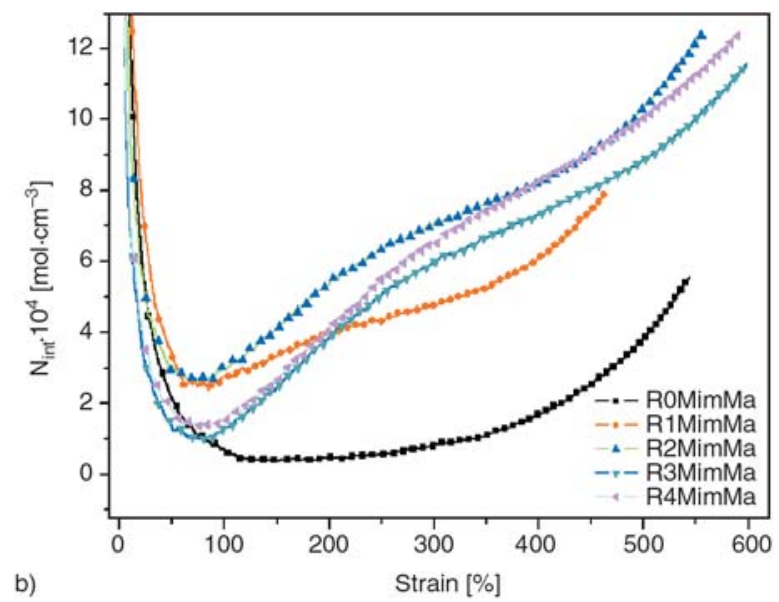

Figure 9. Dependence of stress (a) and $N_{\text {int }}$ (b) on tensile strains 


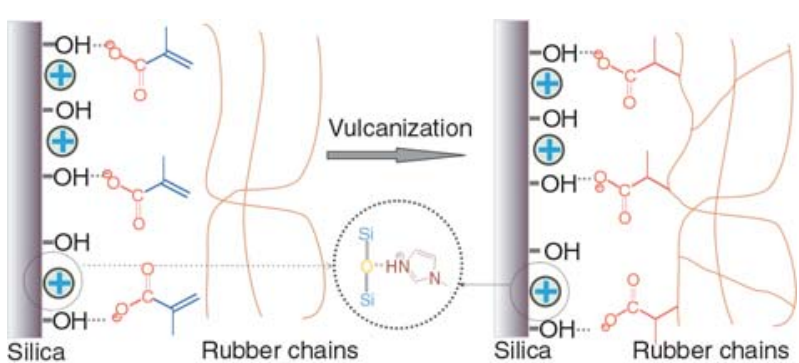

Figure 10. Proposed interfacial structure for the SBR/silica/MimMa composites

restrained by weakening the interactions among silica particles. Once the vulcanization is performed, MimMa is mainly grafted onto SBR molecules via a radical mechanism. The graft product, poly(SBRg-MimMa), can effectively enhance the compatibility between MimMa and SBR. The hydrogen bonding interactions, which exist in both uncured rubber compounds and rubber vulcanizates, can not only contribute the filler dispersion but also the structure change of SBR/silica interface.

\section{Conclusions}

A novel functional ionic liquid (IL), 1-methylimidazolium methacrylate (MimMa), was synthesized and investigated as a modifier in a SBR/silica composites. The affinity of MimMa toward silica was proved to be hydrogen bonding between silica and the ionic units. The interaction among silica particles was weakened and the filler networking of silica in the rubber matrix was effectively restrained. MimMa was found to be reactive toward SBR chains through its graft copolymerization onto rubber chains during vulcanization. With a small addition of MimMa, silica dispersion and mechanical performance of the vulcanizates were effectively improved. The increased mechanical loss under dynamic load indicated a promising utilization as a damping material. A modified interfacial structure, which included the high affinity of MimMa toward silica and its reactivity to rubber chains, was accordingly proposed. Remarkable improvements in the rubber performances were related to the proposed interfacial structure.

\section{Acknowledgements}

The authors thank National Natural Science Foundation of China (50873035, 50933005), Guangdong Natural Science Foundation (151008901000137), Fundamental Research for the Central Universities (200922007) and National High-tech R\&D Program (863 Program; 2009AA03Z338), for their financial supports.

\section{References}

[1] Jesionowski T., Krysztafkiewicz A.: Influence of silane coupling agents on surface properties of precipitated silicas. Applied Surface Science, 172, 18-32 (2001). DOI: $\underline{10.1016 / S 0169-4332(00) 00828-X}$

[2] Liu Y-H., Lin H-P., Mou C-Y.: Direct method for surface silyl functionalization of mesoporous silica. Langmuir, 20, 3231-3239 (2004). DOI: $10.1021 / \mathrm{la} 0358421$

[3] Rao A. V., Kulkarni M. M., Amalnerkar D. P., Seth T.: Surface chemical modification of silica aerogels using various alkyl-alkoxy/chloro silanes. Applied Surface Science, 206, 262-270 (2003). DOI: $\underline{10.1016 / \mathrm{S} 0169-4332(02) 01232-1}$

[4] Wang M. J.: Effect of polymer-filler and filler-filler interactions on dynamic properties of filled vulcanizates. Rubber Chemistry and Technology, 71, 520 589 (1998).

[5] Rogers R. D., Seddon K. R.: Ionic liquids- Solvents of the future? Science, 302, 792-793 (2003).

DOI: $10.1126 /$ science. 1090313

[6] Wasserscheid P., Keim W.: Ionic liquids - New 'solutions' for transition metal catalysis. Angewandte Chemie-International Edition, 39, 3773-3789 (2000). DOI: 10.1002/1521-3773(20001103)39:21<3772:: AID-ANIE3772>3.0.CO;2-5

[7] Huddleston J. G., Visser A. E., Reichert W. M., Willauer H. D., Broker G. A., Rogers R. D.: Characterization and comparison of hydrophilic and hydrophobic room temperature ionic liquids incorporating the imidazolium cation. Green Chemistry, 3, 156-164 (2001).

DOI: $10.1039 / \mathrm{b} 103275 \mathrm{p}$

[8] Fukushima T., Kosaka A., Ishimura Y., Yamamoto T., Takigawa T., Ishii N., Aida T.: Molecular ordering of organic molten salts triggered by single-walled carbon nanotubes. Science, 300, 2072-2074 (2003). DOI: $10.1126 /$ science. 1082289

[9] Turner M. B., Spear S. K., Holbrey J. D., Rogers R. D.: Production of bioactive cellulose films reconstituted from ionic liquids. Biomacromolecules, 5, 1379 1384 (2004). DOI: $\underline{10.1021 / \mathrm{bm} 049748 \mathrm{q}}$ 
[10] Whitten P. G., Spinks G. M., Wallace G. G.: Mechanical properties of carbon nanotube paper in ionic liquid and aqueous electrolytes. Carbon, 43, 1891-1896 (2005).

DOI: $10.1016 /$ j.carbon.2005.02.038

[11] Fukushima T., Asaka K., Kosaka A., Aida T.: Fully plastic actuator through layer-by-layer casting with ionic-liquid-based bucky gel. Angewandte ChemieInternational Edition, 44, 2410-2413 (2005). DOI: $10.1002 / a n i e .200462318$

[12] Zhou X. S., Wu T. B., Ding K. L., Hu B. J., Hou M. Q., Han B. X.: The dispersion of carbon nanotubes in water with the aid of very small amounts of ionic liquid. Chemical Communications, 2009, 1897-1899 (2009). DOI: $10.1039 / \mathrm{b} 900849 \mathrm{~g}$

[13] Wang J. Y., Chu H. B., Li Y.: Why single-walled carbon nanotubes can be dispersed in imidazolium-based ionic liquids. ACS Nano, 2, 2540-2546 (2008). DOI: $10.1021 / \mathrm{nn} 800510 \mathrm{~g}$

[14] Zhang Y. J., Shen Y. F., Li J. H., Niu L., Dong S. J., Ivaska A.: Electrochemical functionalization of single-walled carbon nanotubes in large quantities at a room-temperature ionic liquid supported three-dimensional network electrode. Langmuir, 21, 4797-4800 (2005).

DOI: $10.1021 / \mathrm{la} 050026+$

[15] Gobel R., Hesemann P., Weber J., Moller E., Friedrich A., Beuermann S., Taubert A.: Surprisingly high, bulk liquid-like mobility of silica-confined ionic liquids. Physical Chemistry Chemical Physics, 11, 3653-3662 (2009).

DOI: $10.1039 / \mathrm{b} 821833 \mathrm{a}$

[16] Zhou Y., Schattka J. H., Antonietti M.: Room-temperature ionic liquids as template to monolithic mesoporous silica with wormlike pores via a sol-gel nanocasting technique. Nano Letters, 4, 477-481 (2004). DOI: $\underline{10.1021 / \mathrm{nl} 025861 \mathrm{f}}$

[17] Cammarata L., Kazarian S. G., Salter P. A., Welton T.: Molecular states of water in room temperature ionic liquids. Physical Chemistry Chemical Physics, 3, 5192-5200 (2001). DOI: $10.1039 / \mathrm{b} 106900 \mathrm{~d}$

[18] Byrne C., McNally T.: Ionic liquid modification of layered silicates for enhanced thermal stability. Macromolecular Rapid Communications, 28, 780-784 (2007). DOI: $10.1002 /$ marc. 200600754

[19] Ma J. C., Dougherty D. A.: The cation- $\pi$ interaction. Chemical Reviews, 97, 1303-1324 (1997). DOI: $10.1021 / \mathrm{cr} 9603744$

[20] Yoo K., Choi H., Dionysiou D. D.: Ionic liquid assisted preparation of nanostructured $\mathrm{TiO}_{2}$ particles. Chemical Communications, 2004, 2000-2001 (2004). DOI: $10.1039 / \mathrm{b} 406040 \mathrm{~g}$
[21] Wu B. H., Hu D., Kuang Y. J., Liu B., Zhang X. H., Chen J. H.: Functionalization of carbon nanotubes by an ionic-liquid polymer: Dispersion of Pt and PtRu nanoparticles on carbon nanotubes and their electrocatalytic oxidation of methanol. Angewandte ChemieInternational Edition, 48, 4751-4754 (2009). DOI: 10.1002/anie.200900899

[22] Tu W. W., Lei J. P., Ju H. X.: Functionalization of carbon nanotubes with water-insoluble porphyrin in ionic liquid: Direct electrochemistry and highly sensitive amperometric biosensing for trichloroacetic acid. Chemistry-A European Journal, 15, 779-784 (2009). DOI: $10.1002 /$ chem. 200801758

[23] Chen Y. T., Chen X., Lin Z. Y., Dai H., Qiu B., Sun J. J., Zhang L., Chen G. N.: An electrically heated ionicliquid/multi-wall carbon nanotube composite electrode and its application to electrochemiluminescent detection of ascorbic acid. Electrochemistry Communications, 11, 1142-1145 (2009). DOI: $10.1016 /$ j.elecom.2009.03.033

[24] Yuan L-M., Ren C-X., Li L., Ai P., Yan Z-H., Zi M., Li Z-Y.: Single-walled carbon nanotubes used as stationary phase in GC. Analytical Chemistry, 78, 6384 6390 (2006). DOI: $10.1021 / \mathrm{ac} 060663 \mathrm{k}$

[25] Xu Y-P., Tian Z-J., Wang S-J., Hu Y., Wang L., Wang B-C., Ma Y-C., Hou L., Yu J-Y., Lin L-W.: Microwave-enhanced ionothermal synthesis of aluminophosphate molecular sieves. Angewandte ChemieInternational Edition, 45, 3965-3970 (2006). DOI: $10.1002 /$ anie.200600054

[26] Dai Z., Xiao Y., Yu X. Z., Mai Z. B., Zhao X. J., Zou X. Y.: Direct electrochemistry of myoglobin based on ionic liquid-clay composite films. Biosensors and Bioelectronics, 24, 1629-1634 (2009). DOI: $10.1016 /$ j.bios.2008.08.032

[27] Dupont J., Suarez P. A. Z.: Physico-chemical processes in imidazolium ionic liquids. Physical Chemistry Chemical Physics, 8, 2441-2452 (2006).

DOI: $10.1039 / \mathrm{b} 602046 \mathrm{a}$

[28] Winterton N.: Solubilization of polymers by ionic liquids. Journal of Materials Chemistry, 16, 4281-4293 (2006).

DOI: $10.1039 / \mathrm{b} 610143 \mathrm{~g}$

[29] Marwanta E., Mizumo T., Nakamura N., Ohno H.: Improved ionic conductivity of nitrile rubber/ionic liquid composites. Polymer, 46, 3795-3800 (2005). DOI: 10.1016/j.polymer.2005.02.113

[30] Swatloski R. P., Holbrey J. D., Rogers R. D.: Ionic liquids are not always green: Hydrolysis of 1-butyl-3methylimidazolium hexafluorophosphate. Green Chemistry, 5, 361-363 (2003). DOI: $10.1039 / \mathrm{B} 304400 \mathrm{a}$ 
[31] Lei Y. D., Guo B. C., Liu X. L., Jia D. M.: Structure evolution of carbon black under ionic-liquid-assisted microwave irradiation. Applied Surface Science, 255, 8488-8493 (2009).

DOI: $10.1016 /$ j.apsusc. 2009.05.163

[32] Lei Y. D., Guo B. C., Tang Z. H., Jia D. M.: SBR/silica composites modified by a polymerizable protic ionic liquid. Polymer Journal, 42, 555-561 (2010). DOI: $10.1038 /$ pj.2010.43

[33] Guo B., Liu X., Zhou W. Y., Lei Y. D., Jia D. M.: Adsorption of ionic liquid onto halloysite nanotubes: Mechanism and reinforcement of the modified clay to rubber. Journal of Macromolecular Science Part B: Physics, 49, 1029-1043 (2010). DOI: $10.1080 / 00222341003609823$

[34] Belieres J-P., Angell C. A.: Protic ionic liquids: Preparation, characterization, and proton free energy level representation. Journal of Physical Chemistry B, 111, 4926-4937 (2007).

DOI: $10.1021 / \mathrm{jp} 067589 \mathrm{u}$

[35] Greaves T. L., Weerawardena A., Fong C., Drummond C. J.: Many protic ionic liquids mediate hydrocarbon-solvent interactions and promote amphiphile self-assembly. Langmuir, 23, 402-404 (2006). DOI: $10.1021 / \mathrm{la} 062895 \mathrm{k}$

[36] Lin B. C., Cheng S., Qiu L. H., Yan F., Shang S. M., Lu J. M.: Protic ionic liquid-based hybrid proton-conducting membranes for anhydrous proton exchange membrane application. Chemistry of Materials, 22, 1807-1813 (2010). DOI: $10.1021 / \mathrm{Cm} 9033758$

[37] Nakamoto H., Watanabe M.: Brønsted acid-base ionic liquids for fuel cell electrolytes. Chemical Communications, 2007, 2539-2541 (2007).

DOI: $10.1039 / \mathrm{b} 618953 \mathrm{a}$

[38] Huang M-M., Weingärtner H.: Protic ionic liquids with unusually high dielectric permittivities. ChemPhysChem, 9, 2172-2173 (2008).

DOI: $10.1002 / \mathrm{cphc} .200800523$

[39] Guo B. C., Chen F., Lei Y. D., Liu X. L., Wan J. J., Jia D. M.: Styrene-butadiene rubber/halloysite nanotubes nanocomposites modified by sorbic acid. Applied Surface Science, 255, 7329-7336 (2009). DOI: $10.1016 /$ j.apsusc. 2009.03 .092

[40] Rajkumar T., Rao G. R.: Synthesis and characterization of hybrid molecular material prepared by ionic liquid and silicotungstic acid. Materials Chemistry and Physics, 112, 853-857 (2008). DOI: $10.1016 /$ j.matchemphys.2008.06.046

[41] Jerman I., Jovanovski V., Vuk A. Š., Hocevar S. B., Gaberšcek M., Jesih A., Orel B.: Ionic conductivity, infrared and Raman spectroscopic studies of 1-methyl3-propylimidazolium iodide ionic liquid with added iodine. Electrochimica Acta, 53, 2281-2288 (2008). DOI: $10.1016 /$ j.electacta.2007.09.043
[42] Vuk A. Š., Jovanovski V., Pollet-Villard A., Jerman I., Orel B.: Imidazolium-based ionic liquid derivatives for application in electrochromic devices. Solar Energy Materials and Solar Cells, 92, 126-135 (2008).

DOI: $10.1016 /$ j.solmat.2007.01.023

[43] Yu J. J., Ryu S. H.: Ultraviolet-initiated photografting of glycidyl methacrylate onto styrene-butadiene rubber. Journal of Applied Polymer Science, 73, 1733 1739 (1999).

DOI: 10.1002/(SICI)1097-4628(19990829)73:9<1733 $\because$ AID-APP14>3.0.CO;2-J

[44] Watts J. F., Wolstenholme J.: An introduction to surface analysis by XPS and AES. Wiley, Chichester (2003). DOI: $\underline{10.1002 / 0470867930}$

[45] Romero-Sánchez M. D., Pastor-Blas M. M., MartínMartínez J. M.: Adhesion improvement of SBR rubber by treatment with trichloroisocyanuric acid solutions in different esters. International Journal of Adhesion and Adhesives, 21, 325-337 (2001).

DOI: $\underline{10.1016 / \mathrm{S} 0143-7496(01) 00005-7}$

[46] Paparazzo E.: On the XPS analysis of $\mathrm{Si}-\mathrm{OH}$ groups at the surface of silica. Surface and Interface Analysis, 24, 729-730 (1996).

DOI: 10.1002/(SICI)1096-9918(19960930)24:10<729 $\because$ AID-SIA183>3.0.CO;2-P

[47] Wang M-J.: The role of filler networking in dynamic properties of filled rubber. Rubber Chemistry and Technology, 72, 430-448 (1999).

[48] Payne A. R.: The dynamic properties of carbon blackloaded natural rubber vulcanizates. Part I. Journal of Applied Polymer Science, 6, 57-63 (1962).

DOI: $\underline{10.1002 / a p p .1962 .070061906}$

[49] Murphy L. J., Wang M. J., Mahmud K.: Carbon-silica dual phase filler: Part III. ESCA and IR characterization. Rubber Chemistry and Technology, 71, 998 1014 (1998).

[50] Murphy L. J., Khmelnitskaia E., Wang M-J., Mahmud K.: Carbon-silica dual phase filler: Part IV. Surface chemistry. Rubber Chemistry and Technology, 71, 1015-1027 (1998).

[51] Wang M. J.: Effect of filler-elastomer interaction on tire tread performance part II. Kautschuk Gummi Kunststoffe, 61, 33-42 (2008).

[52] Wang M. J., Mahmud K., Murphy L. J., Patterson W. J.: Carbon-silica dual phase filler, a new generation reinforcing agent for rubber, Part I. Characterization. Kautschuk Gummi Kunststoffe, 51, 348-359 (1998).

[53] Wang M-J., Lu S. X., Mahmud K.: Carbon-silica dualphase filler, a new-generation reinforcing agent for rubber. Part VI. Time-temperature superposition of dynamic properties of carbon-silica-dual-phase-fillerfilled vulcanizates. Journal of Polymer Science Part B: Polymer Physics, 38, 1240-1249 (2000). DOI: $10.1002 /($ SICI $1099-0488(20000501) 38: 9<1240$ $\because$ AID-POLB15>3.0.CO;2-Q 
[54] Wang M. J.: Effect of filler-elastomer interaction on tire tread performance. Part III. Kautschuk Gummi Kunststoffe, 61, 159-165 (2008).

[55] Ayala J. A., Hess W. M., Dotson A. O., Joyce G. A.: New studies on the surface properties of carbon blacks. Rubber Chemistry and Technology, 63, 747-778 (1990).

[56] Ayala J. A., Hess W. M., Kistler F. D., Joyce G. A.: Carbon-black-elastomer interaction. Rubber Chemistry and Technology, 64, 19-39 (1991).

[57] Zhang H., Datta R. N., Talma A. G., Noordermeer J. W. M.: Modification of EPDM with alkylphenol polysulfide for use in tire sidewalls, 2-mechanistic and morphological characterizations. Macromolecular Materials and Engineering, 295, 76-83 (2010).

DOI: $10.1002 / \mathrm{mame} .200900126$
[58] Flory P. J., Rehner J.: Statistical mechanics of crosslinked polymer networks I. Rubberlike elasticity. The Journal of Chemical Physics, 11, 512-520 (1943). DOI: $10.1063 / 1.1723791$

[59] Flory P. J., Rehner J.: Statistical mechanics of crosslinked polymer networks II. Swelling. The Journal of Chemical Physics, 11, 521-526 (1943). DOI: $\underline{10.1063 / 1.1723792}$

[60] Cai J. J., Salovey R.: Model filled rubber, Part V. Mechanical properties of rubbery composites. Journal of Materials Science, 36, 3947-3953 (2001). DOI: $\underline{10.1023 / \mathrm{A}: 1017922322114}$ 